Appl. Set-Valued Anal. Optim. 2 (2020), No. 1, pp. 123-137

Available online at http://asvao.biemdas.com

https://doi.org/10.23952/asvao.2.2020.1.08

\title{
ON GENERALIZED $\tau^{w}$-CONTINGENT EPIDERIVATIVES IN PARAMETRIC VECTOR OPTIMIZATION PROBLEMS
}

\author{
LE THANH TUNG ${ }^{1, *}$, PHAM THANH HUNG ${ }^{2}$ \\ ${ }^{1}$ Department of Mathematics, College of Natural Sciences, Can Tho University, Can Tho, Vietnam \\ ${ }^{2}$ Faculty of Pedagogy and Faculty of Social Sciences \& Humanities, Kien Giang University, Kien Giang, Vietnam
}

\begin{abstract}
This paper is concerned with the sensitivity analysis in reflexive Banach spaces. We first establish the formulae for estimating the generalized $\tau^{w}$-contingent epiderivatives of the efficient solution maps and the perturbation maps in parametric vector optimization problems. Then, an application to the semi-infinite programming is provided. Some examples are also given for illustrating the obtained results.
\end{abstract}

Keywords. Parametric vector optimization problems; Generalized $\tau^{w}$-contingent epiderivatives; Efficient solution maps; Perturbation maps; Semi-infinite inequality constraints.

\section{INTRODUCTION}

In parametric vector optimization problems, sensitivity analysis is the research of derivatives of the perturbation maps and the efficient solution maps. There are two main approaches in sensitivity analysis: the dual space approach and the primal space approach. In the dual space approach, we refer to the books $[16,17]$ and the recent papers $[6,8,18]$. In primal space approach, the first-order derivatives of perturbation maps were studied in $[3,5,7,13,19,26$, 33]. To get more information, the higher-order derivatives of perturbation maps have been investigated in $[1,9,27,29]$. Another interesting topic in the primal space approach is to study the proto-differentiability of perturbation maps, introduced in [21]. Some developments on the proto-differentiability of perturbation maps of parametric vector optimization problems and related problems were obtained in $[11,14,15,30,31]$.

In [22], $\tau^{w}$-contingent epiderivatives, based on the consideration of the weak topology in the image space, were introduced, and applied to the optimality conditions for a set-valued optimization problem. The formulas for estimating the $\tau^{w}$-contingent derivative of the Borwein proper perturbation map via the $\tau^{w}$-contingent of the constraint map and the Hadamard derivative of the objective map were considered in [33]. It should be noted that contingent epiderivatives [12] as well as $\tau^{w}$-contingent epiderivatives may not exist in some cases. To deal with this problem, an alternative definition of contingent epiderivative, denoted by generalized contingent epiderivatives, for a set-valued map was presented in $[3,4]$. We observe that this alternative definition could be done in the same way for $\tau^{w}$-contingent epiderivatives. Moreover, the generalized $\tau^{w}$-contingent epiderivatives are able to utilize in sensitivity analysis.

${ }^{*}$ Corresponding author.

E-mail addresses: Ittung@ctu.edu.vn (L.T. Tung), pthung@ @nkgu.edu.vn (P.T. Hung).

Received January 27, 2020; Accepted March 2, 2020.

(C)2020 Applied Set-Valued Analysis and Optimization 
Motivated by the above observations, we aim to discuss the generalized $\tau^{w}$-contingent derivatives in sensitivity analysis by considering the generalized $\tau^{w}$-contingent derivatives of the efficient solution maps and the perturbation maps of parametric vector optimization problems in this paper. The organization of the paper is as follows. In Section 2, we recall some important notions and present the definition of the generalized $\tau^{w}$-contingent epiderivatives of set-valued maps. In Section 3, we establish the formulae for computing the generalized $\tau^{w}$-contingent epiderivative of the efficient solution map and the pertubation map of a parametric vector optimization problem. An application to semi-infinite optimization is given in Section 4.

\section{PRELIMINARIES}

Throughout this paper, let $P, X$ and $Y$ be reflexive Banach spaces, where the spaces $X$ and $Y$ are partially ordered by pointed, closed, and convex cones with apex at the origin $Q \subseteq X$ and $K \subseteq$ $Y$, respectively (shortly resp). The norm of Banach space $P \times Y$ are defined by $\|(p, y)\|_{P \times Y}=$ $\|p\|_{P}+\|y\|_{Y}$. For $A \subseteq Y$, int $A, \operatorname{cl} A, \partial A$, cone $A$ denote its interior, closure, boundary and the cone $\{\lambda a \mid \lambda \geq 0, a \in A\}$, resp. A set $B \subset Y$ is called a base for $K$ if $0 \notin \operatorname{cl} B$ and $K=\{\lambda b$ : $\lambda>0, b \in B\}$. If $B$ is compact we say that $K$ has a compact base $B$. The cone $K$ has a compact base if and only if $K \cap \partial B$ is compact (see in [26]). The set of all neighborhoods of $y \in Y$ is represented by $\mathscr{N}(y)$.

Let $A$ be a nonempty subset of $Y$ and $\bar{a} \in A$. We recall some concepts of optimality/efficiency in vector optimization as follows.

(i) $\bar{a}$ is called a local (Pareto) minimal/efficient point of $A$ with respect to (shortly wrt) $K$, denoted by $\bar{a} \in \operatorname{Min}_{K} A$ iff there exists $U \in \mathscr{U}(\bar{a})$ such that

$$
(A \cap U-\bar{a}) \cap(-K \backslash\{0\})=\emptyset .
$$

(ii) $\bar{a}$ is said to be a local weak minimal/efficient point of $A$, denoted by $a \in \operatorname{WMin}_{K} A$ iff there exists $U \in \mathscr{U}(\bar{a})$ such that

$$
(A \cap U-\bar{a}) \cap(-\operatorname{int} K)=\emptyset .
$$

(iii) $\bar{a}$ is said to be a local ideal minimal/efficient point of $A$, denoted by $a \in \operatorname{IMin}_{K} A$ iff there exists $U \in \mathscr{U}(\bar{a})$ such that

$$
A \cap U-\bar{a} \subset K .
$$

If $U=Y$, the word "local" is omitted, i.e., we have the corresponding global notions. It is easy to see that $\operatorname{IMin}_{K} A \subseteq \operatorname{Min}_{K} A \subseteq \mathrm{WMin}_{K} A$. Note that $\operatorname{IMin}_{K} A$ may be not a single-point set when $K$ is not pointed. Moreover, $\operatorname{Min}_{K}(A+K)=\operatorname{Min}_{K} A$, see [25].

Let $G: P \rightrightarrows Y$ be a set-valued map. The effective domain, graph and epigraph of $G$ is defined resp by

$$
\begin{array}{ll}
\operatorname{dom} G & :=\{p \in P \mid G(p) \neq \emptyset\}, \\
\operatorname{gr} G & :=\{(p, y) \in P \times Y \mid y \in G(p)\}, \\
\operatorname{epi} G & :=\{(p, y) \in P \times Y \mid p \in \operatorname{dom} G, y \in G(p)+K\} .
\end{array}
$$

The so-called profile map $G+K: P \rightrightarrows Y$ is defined by $(G+K)(p):=G(p)+K$. In the sequel, by $\rightarrow$ (resp. $\rightarrow$ ) we denote the convergence wrt the norm topology (resp. weak topology). Given $\left(p_{n}, y_{n}\right) \in P \times Y$ and $(\bar{p}, \bar{y}) \in P \times Y$, by $\left(p_{n}, y_{n}\right) \underset{s, w}{\longrightarrow}(\bar{p}, \bar{y})$, we mean $p_{n} \rightarrow \bar{p}, y_{n} \underset{w}{\rightarrow} \bar{y}$.

Definition 2.1. [22] Let $M \subset P \times Y$ and $(\bar{p}, \bar{y}) \in M$. 
(i) The weak contingent cone of $M$ at $(\bar{p}, \bar{y})$ is defined by

$$
T^{w}(M,(\bar{p}, \bar{y})):=\left\{(p, y) \in P \times Y \mid \exists t_{n} \downarrow 0, \exists\left(p_{n}, y_{n}\right) \underset{s, w}{\longrightarrow}(p, y),(\bar{p}, \bar{y})+t_{n}\left(p_{n}, y_{n}\right) \in M, \forall n\right\}
$$

(ii) The $\tau^{w}$-contingent derivative of a set-valued map $G: P \rightrightarrows Y$ at $(\bar{p}, \bar{y}) \in \operatorname{gr} G$ is the setvalued map $D^{w} G(\bar{p}, \bar{y}): P \rightrightarrows Y$ such that $\operatorname{gr} D^{w} G(\bar{p}, \bar{y})=T^{w}(\operatorname{gr} G,(\bar{p}, \bar{y}))$, or equivalently, $D^{w} G(\bar{p}, \bar{y})(p):=\left\{y \in Y \mid \exists t_{n} \downarrow 0, \exists\left(p_{n}, y_{n}\right) \underset{s, w}{\longrightarrow}(p, y), \bar{y}+t_{n} y_{n} \in G\left(\bar{p}+t_{n} p_{n}\right), \forall n\right\}$.

(iii) The set-valued $G$ is said to be $\tau^{w}$-epidifferentiable at $(\bar{p}, \bar{y}) \in \mathrm{grG}$ if there exists a singlevalued map $E D^{w} G(\bar{p}, \bar{y}): \operatorname{dom} D^{w}(G+K)(\bar{p}, \bar{y}) \subseteq P \rightarrow Y$ such that

$$
\operatorname{epi} E D^{w} G(\bar{p}, \bar{y})=T^{w}(\text { epi } G,(\bar{p}, \bar{y})) \text {. }
$$

Then, $E D^{w} G(\bar{p}, \bar{y})$ is called $\tau^{w}$-contingent epiderivative of $G$ at $(\bar{p}, \bar{y})$.

Remark 2.1. [22] If $G$ is be $\tau^{w}$-epidifferentiable at $(\bar{p}, \bar{y}) \in \operatorname{grG}$, then

$$
E D^{w} G(\bar{p}, \bar{y})(p)=\operatorname{IMin}_{K} D^{w}(G+K)(\bar{p}, \bar{y})(p)
$$

for all $p \in \operatorname{dom} D^{w}(G+K)(\bar{p}, \bar{y})$.

Lemma 2.1. [33] Let $G: P \rightrightarrows Y$ and $(\bar{p}, \bar{y}) \in \operatorname{gr} G$. Then,

$$
D^{w} G(\bar{p}, \bar{y})(p)+K \subseteq D^{w}(G+K)(\bar{p}, \bar{y})(p) .
$$

In the line of $[3,4]$, we introduce the following definition of generalized $\tau^{w}$-contingent epiderivative of a set-valued map.

Definition 2.2. The generalized $\tau^{w}$-contingent epiderivative of a set-valued map $G: P \rightrightarrows Y$ at $(\bar{p}, \bar{y}) \in \operatorname{gr} G$ is the set-valued map $D_{g}^{w} G(\bar{p}, \bar{y}): P \rightrightarrows Y$ defined by

$$
D_{g}^{w} G(\bar{p}, \bar{y})(p):=\operatorname{Min}_{K} D^{w}(G+K)(\bar{p}, \bar{y})(p), \forall p \in P .
$$

Example 2.1. [24] Let $P=\mathbb{R}$ and $Y=l^{2}$, where $l^{2}$ is the space of all scalar sequences $x=$ $\left\{x_{i}\right\}_{i \in \mathbb{N}}=\left(x_{1}, x_{2}, \ldots, x_{n}, \ldots\right), x_{i} \in \mathbb{R}$ with $\sum_{i=1}^{\infty}\left|x_{i}\right|^{2}<+\infty$ and $\|x\|=\left(\sum_{i=1}^{\infty}\left|x_{i}\right|^{2}\right)^{1 / 2}$. By $\left\{e_{i}\right\}_{i \in \mathbb{N}} \subset$ $l^{2}$ we indicate its standard unit basis. We note the ordering cone on $l^{2}$ as follows

$$
K=l_{+}^{2}=\left\{y=\left\{y_{i}\right\}_{i \in \mathbb{N}} \in l^{2} \mid y_{i} \geq 0 \text { for every } i \in \mathbb{N}\right\} .
$$

$K$ is a closed, convex, and pointed cone with $\operatorname{int} K=\emptyset$. Let the map $G: P \rightarrow Y$ be defined by

$$
G(p)=\left\{\begin{array}{cl}
p\left(h+e_{n}\right), & \text { if } p=\frac{1}{3 n}, \\
p\left(l+e_{n}\right), & \text { if } p=\frac{1}{3 n+1}, \\
p r, & \text { if } p=\frac{1}{3 n+2}, \\
p t, & \text { elsewhere in } \mathbb{R}_{+},
\end{array}\right.
$$

where $h, l, r, t \in l^{2}$ and $(\bar{p}, \bar{y})=\left(0,0_{l^{2}}\right) \in \operatorname{gr} G$.

* Case 1. If $h=l=0, r=t=e_{1}$, then

$$
E D^{w} G(\bar{p}, \bar{y})(p)=D_{g}^{w} G(\bar{p}, \bar{y})(p)=\left\{0_{l^{2}}\right\}, \forall p \in \mathbb{R}_{+} .
$$

* Case 2. If $h=l=e_{1}, r=t=e_{2}$, then $E D^{w} G(\bar{p}, \bar{y})(p)$ does not exists and

$$
D_{g}^{w} G(\bar{p}, \bar{y})(p)=\left\{p e_{1}, p e_{2}\right\}, \forall p \in \mathbb{R}_{+} .
$$


Example 2.2. Let $P=\mathbb{R}$ and $Y=l^{2} \times l^{2}$, where $l^{2}$ is the space of all scalar sequences $x=$ $\left\{x_{i}\right\}_{i \in \mathbb{N}} \subset \mathbb{R}$ with $\sum_{i=1}^{\infty}\left|x_{i}\right|^{2}<+\infty$. Note that $Y$ is also a Hilbert space with the inner product

$$
\left\langle(y, z),\left(y^{\prime}, z^{\prime}\right)\right\rangle_{Y}=\left\langle y, y^{\prime}\right\rangle_{l^{2}}+\left\langle z, z^{\prime}\right\rangle_{l^{2}} \text {. }
$$

By $\left\{e_{i}\right\}_{i \in \mathbb{N}} \subset l^{2}$, we indicate its standard unit basis. We note the ordering cone on $Y$ as follows

$$
K=\left\{(y, z) \in l^{2} \times l^{2} \mid y_{i} \geq 0, z_{i} \geq 0 \text { for every } i \in \mathbb{N}\right\} .
$$

$K$ is a closed, convex, and pointed cone with $\operatorname{int} K=\emptyset$. Let the set-valued map $G: P \rightrightarrows Y$ be defined by

$$
G(p)=\left\{\begin{array}{cl}
\left\{(y, z) \in Y \mid\|(y, z)\|_{Y}^{2} \leq p^{2}\right\}, & \text { if } 0 \leq p \leq 1 \\
\emptyset, & \text { otherwise }
\end{array}\right.
$$

and

$$
(\bar{p},(\bar{y}, \bar{z}))=\left(1,\left(\left(-\frac{1}{\sqrt{2}}, 0, \ldots, 0, \ldots\right),\left(-\frac{1}{\sqrt{2}}, 0, \ldots, 0, \ldots\right)\right) \in \operatorname{gr} G\right.
$$

Then,

$$
T^{w}(\operatorname{gr} G,(\bar{p},(\bar{y}, \bar{z})))=\left\{(p,(y, z)) \in P \times Y \mid y_{1}+z_{1} \geq-\sqrt{2} p\right\} .
$$

Indeed, let

$$
((p,(y, z))) \in T^{w}(\operatorname{gr} G,(\bar{p},(\bar{y}, \bar{z}))) .
$$

Then, there exist $t_{n} \downarrow 0,\left(p_{n},\left(y^{(n)}, z^{(n)}\right)\right) \underset{s, w}{\longrightarrow}(p,(y, z))$ such that

$$
\left(1,\left(\left(-\frac{1}{\sqrt{2}}, 0, \ldots, 0, \ldots\right),\left(-\frac{1}{\sqrt{2}}, 0, \ldots, 0, \ldots\right)\right)+t_{n}\left(p_{n},\left(y^{(n)}, z^{(n)}\right)\right) \in \operatorname{gr} G, \forall n,\right.
$$

leads to

$$
\left\|\left(\left(-\frac{1}{\sqrt{2}}, 0, \ldots, 0, \ldots\right),\left(-\frac{1}{\sqrt{2}}, 0, \ldots, 0, \ldots\right)+t_{n}\left(y^{(n)}, z^{(n)}\right)\right)\right\|_{Y}^{2} \leq\left(1+t_{n} p_{n}\right)^{2}, \forall n .
$$

Consequently,

$$
\begin{aligned}
& \left\langle\left(-\frac{1}{\sqrt{2}}, 0, \ldots, 0, \ldots\right)+t_{n} y^{(n)},\left(-\frac{1}{\sqrt{2}}, 0, \ldots, 0, \ldots\right)+t_{n} y^{(n)}\right\rangle \\
& +\left\langle\left(-\frac{1}{\sqrt{2}}, 0, \ldots, 0, \ldots\right)+t_{n} z^{(n)},\left(-\frac{1}{\sqrt{2}}, 0, \ldots, 0, \ldots\right)+t_{n} z^{(n)}\right\rangle \\
& \leq 1+2 t_{n} p_{n}+t_{n}^{2} p_{n}^{2},
\end{aligned}
$$

or equivalently,

$$
\begin{aligned}
& 2\left\langle\left(-\frac{1}{\sqrt{2}}, 0, \ldots, 0, \ldots\right),\left(-\frac{1}{\sqrt{2}}, 0, \ldots, 0, \ldots\right)\right\rangle \\
& +2 t_{n}\left\langle\left(-\frac{1}{\sqrt{2}}, 0, \ldots, 0, \ldots\right), y^{(n)}+z^{(n)}\right\rangle+t_{n}^{2}\left(\left\langle y^{(n)}, y^{(n)}\right\rangle+\left\langle z^{(n)}, z^{(n)}\right\rangle\right) \\
& \leq 1+2 t_{n} p_{n}+t_{n}^{2} p_{n}^{2} .
\end{aligned}
$$

Hence,

$$
-2 \cdot \frac{1}{\sqrt{2}}\left(y_{1}^{(n)}+z_{1}^{(n)}\right)+t_{n}\left(\left\langle y^{(n)}, y^{(n)}\right\rangle+\left\langle z^{(n)}, z^{(n)}\right\rangle\right) \leq 2 p_{n}+t_{n} p_{n}^{2}, \forall n .
$$

Letting $n \rightarrow \infty$, one has $y_{1}+z_{1} \geq-\sqrt{2} p$. Moreover, we also have

$$
T^{w}(\operatorname{epi} G,(\bar{p},(\bar{y}, \bar{z})))=T^{w}(\operatorname{gr} G,(\bar{p},(\bar{y}, \bar{z})))=\left\{(p,(y, z)) \in P \times Y \mid y_{1}+z_{1} \geq-\sqrt{2} p\right\} .
$$


Hence, $\left.E D^{w} G(\bar{p},(\bar{y}, \bar{z}))\right)$ does not exist and

$$
\left.D_{g}^{w}(\bar{p},(\bar{y}, \bar{z}))\right)(p)=\left\{(y, z) \in Y \mid y_{1}+z_{1}=-\sqrt{2} p\right\} .
$$

Definition 2.3. [25] Let $A \subset Y, G: P \rightrightarrows Y$ and $\bar{p} \in P$.

(i) The set $A$ is said to have the domination property if $A \subset \operatorname{Min}_{K} A+K$.

(ii) We say that the domination property satisfies for $G$ around $\bar{p}$ if there exists a neighborhood $U \in \mathscr{N}(\bar{p})$ such that

$$
G(p) \subset \operatorname{Min}_{K} G(p)+K, \forall p \in U .
$$

Definition 2.4. [2] Let $G: P \rightarrow Y$ be a vector-valued map. $G$ is said to be Fréchet differentiable at $\bar{p} \in P$ iff there exist a linear continuous operator $\nabla G(\bar{p}): P \rightarrow Y$ such that

$$
G(p)=G(\bar{p})+\nabla G(\bar{p})(p-\bar{p})+o(\|p-\bar{p}\|),
$$

where $o(\|p-\bar{p}\|)$ satisfies $\frac{o(\|p-\bar{p}\|)}{\|p-\bar{p}\|} \rightarrow 0$ when $p \rightarrow \bar{p} . \nabla G(\bar{p})$ is called the Fréchet derivative. $G$ is said to be Fréchet differentiable on $P$ if $G$ is Fréchet differentiable at any $p \in P$. If $\nabla G($.$) is continuous at \bar{p}$ then $P$ is said to be continuously Fréchet differentiable at $\bar{p}$.

Definition 2.5. [33] Let $G: P \rightrightarrows Y$ and $(\bar{p}, \bar{y}) \in \operatorname{gr} G$.

(i) The weak radial-contingent cone of $G$ at $(\bar{p}, \bar{y})$, denoted by $T_{S}^{w}(\operatorname{gr} G ;(\bar{p}, \bar{y}))$, is defined by

$$
\begin{aligned}
T_{S}^{w}(\operatorname{gr} G ;(\bar{p}, \bar{y})):= & \left\{(p, y) \in P \times Y \mid \exists t_{n}>0, \exists\left(p_{n}, y_{n}\right) \in \operatorname{gr} G\right. \\
& \text { such that } \left.\left(p_{n}, y_{n}\right) \underset{s, w}{\longrightarrow}(\bar{p}, \bar{y}), \text { with } \bar{y}+t_{n} y_{n} \in G\left(\bar{p}+t_{n} p_{n}\right), \forall n, t_{n} p_{n} \rightarrow 0\right\} .
\end{aligned}
$$

(ii) The $\tau^{w}$-TP-derivative of a set-valued map $G: P \rightrightarrows Y$ at $(\bar{p}, \bar{y})$ is the set-valued map $D_{S}^{w} G(\bar{p}, \bar{y}): P \rightrightarrows Y$ such that

$$
\operatorname{gr}\left(D_{S}^{w} G(\bar{p}, \bar{y})\right)=T_{S}^{w}(\operatorname{gr} G ;(\bar{p}, \bar{y})) .
$$

Definition 2.6. [33] $G$ is called weak directional compact at $(\bar{p}, \bar{y}) \in \operatorname{gr} G$ in the direction $p \in P$ if for every sequence $t_{n} \downarrow 0$ and for any sequence $p_{n} \rightarrow p \in P$, any sequence $y_{n}$ in $Y$ with $\bar{y}+t_{n} y_{n} \in$ $G\left(\bar{p}+t_{n} p_{n}\right)$ for each $n$ includes a weak convergent subsequence. If $G$ is weak directional compact at $(\bar{p}, \bar{y})$ for every $p \in P$, then $G$ is said to be weak directional compact at $(\bar{p}, \bar{y})$.

Lemma 2.2. Let $G: P \rightrightarrows Y$ and $(\bar{p}, \bar{y}) \in \operatorname{gr} G$.

(i) [3] If $G$ is single-valued and Fréchet differentiable at $\bar{p}$, then $G$ is weak directional compact at $(\bar{p}, G(\bar{p}))$.

(ii) [33] If $Y$ is finite dimensional and $D_{S}^{w} G(\bar{p}, \bar{y})(0)=\{0\}$, then $G$ is weak directional compact at $(\bar{p}, \bar{y})$.

Definition 2.7. [20] Let $G: P \rightarrow Y$ be a vector-valued function. $G$ is said to be monotone iff, for any $p_{1}, p_{2} \in P,\left\langle G\left(p_{2}\right)-G\left(p_{1}\right), p_{2}-p_{1}\right\rangle \geq 0$. $G$ is said to be strictly monotone iff, for any $p_{1}, p_{2} \in P$, and $p_{1} \neq p_{2},\left\langle G\left(p_{2}\right)-G\left(p_{1}\right), p_{2}-p_{1}\right\rangle>0$. 


\section{THE GENERALIZED $\tau^{w}$ - CONTINGENT EPIDERIVATIVE OF THE EFFICIENT SOLUTION MAPS AND THE PERTURBATION MAPS}

This paper deals with the sensitivity analysis of parameterized vector optimization problems. First, some notations and definitions are recollected. Let $f: P \times X \rightarrow Y$ be a vector function and $C: P \rightrightarrows X$ be a set-valued map. Let $F: P \rightrightarrows Y$ be a set-valued map defined by

$$
F(p):=f(p, C(p))=\{y \in Y \mid \exists x \in C(p), y=f(p, x)\} .
$$

We consider the following parametric vector optimization problem

$$
\left(\mathrm{PVO}_{p}\right) \quad \operatorname{Min}_{K}\{y \in Y \mid \exists x \in C(p), y=f(p, x)\}=\operatorname{Min}_{K} F(p),
$$

where $x$ is decision variable, $p$ is perturbation parameter, $f$ is objective map, $C$ is constraint map and $F$ is feasible set map in objective spaces. The perturbation (frontier) map $\mathscr{F}: P \rightrightarrows Y$ of a family of parameterized vector optimization problems is defined by

$$
\mathscr{F}(p):=\operatorname{Min}_{K}\{y \in Y \mid \exists x \in C(p), y=f(p, x)\}=\operatorname{Min}_{K} F(p),
$$

and the efficient solution map $\mathscr{S}$ is given by

$$
\mathscr{S}(p):=\{x \in X \mid x \in C(p), f(p, x) \in \mathscr{F}(p)\} .
$$

Proposition 3.1. Let $\bar{p} \in P, \bar{x} \in \mathscr{S}(\bar{p})$ and $\bar{y}=f(\bar{p}, \bar{x})$. Suppose that the following conditions hold:

(i) $f$ is continuously Fréchet differentiable at $(\bar{p}, \bar{x})$ with the derivative $\nabla f(\bar{p}, \bar{x})=\left(\nabla_{p} f(\bar{p}, \bar{x})\right.$, $\left.\nabla_{x} f(\bar{p}, \bar{x})\right)$

(ii) $\nabla_{x} f(\bar{p}, \bar{x})($.$) is strictly monotone on X$;

(iii) $C$ is directionally compact at $(\bar{p}, \bar{x})$ in any direction $p \in X$.

Then,

$$
D_{g}^{w} \mathscr{S}(\bar{p}, \bar{x})(p)=\operatorname{Min}_{Q}\left\{x \in X \mid x \in D^{w} C(\bar{p}, \bar{x})(p), \nabla f(\bar{p}, \bar{x})(p, x) \in D^{w} \mathscr{F}(\bar{p}, \bar{y})(p)\right\}, \forall p \in P .
$$

Proof. First, we prove the following claim, $\forall p \in P$,

$$
D^{w}(\mathscr{S}+Q)(\bar{p}, \bar{x})(p)=\left\{x \in X \mid x \in D^{w} C(\bar{p}, \bar{x})(p), \nabla f(\bar{p}, \bar{x})(p, x) \in D^{w} \mathscr{F}(\bar{p}, \bar{y})(p)\right\}+Q .
$$

Let $x \in D^{w}(\mathscr{S}+Q)(\bar{p}, \bar{x})(p)$. Then, there exist $t_{n} \downarrow 0$ and $\left(p_{n}, x_{n}\right) \underset{s, w}{\longrightarrow}(p, x)$ such that

$$
\bar{x}+t_{n} x_{n} \in \mathscr{S}\left(\bar{p}+t_{n} p_{n}\right)+Q .
$$

This ensures the existence of $\widetilde{x}_{n} \in \mathscr{S}\left(\bar{p}+t_{n} p_{n}\right)$ and $\left\{q_{n}\right\} \subseteq Q$ such that

$$
\bar{x}+t_{n} x_{n}=\widetilde{x}_{n}+q_{n}
$$

which in turn implies that

$$
\widetilde{x}_{n} \in C\left(\bar{p}+t_{n} p_{n}\right) \text { and } f\left(\bar{p}+t_{n} p_{n}, \widetilde{x}_{n}\right) \in \mathscr{F}\left(\bar{p}+t_{n} p_{n}\right) .
$$

Setting $\widetilde{x}_{n}:=\frac{1}{n}\left(\widetilde{x}_{n}-\bar{x}\right)$, we have

$$
\widetilde{x}_{n}=\bar{x}+t_{n} \widetilde{x}_{n}^{\prime} \in C\left(\bar{p}+t_{n} p_{n}\right) \text { and } f\left(\bar{p}+t_{n} p_{n}, \bar{x}+t_{n} \widetilde{x}_{n}^{\prime}\right) \in \mathscr{F}\left(\bar{p}+t_{n} p_{n}\right) .
$$


This together with assumption (iii) implies that $\widetilde{x}_{n}$ has a weak convergent subsequence, denoted also by $\widetilde{x}_{n}$, and $\widetilde{x}_{n} \underset{w}{\rightarrow} \widetilde{x}$, leads to $\widetilde{x}^{\prime} \in D^{w} C(\bar{p}, \bar{x})(p)$. Since $\bar{x}+t_{n} x_{n}=\bar{x}+t_{n} \widetilde{x}_{n}+q_{n}, x_{n} \underset{w}{\rightarrow} x$ and $\tilde{x}_{n} \underset{w}{\rightarrow} \tilde{x}^{\prime}$, one has

$$
x_{n}-\widetilde{x}_{n}=\frac{q_{n}}{t_{n}} \in Q \text { and } x_{n}-\widetilde{x}_{n} \underset{w}{\rightarrow} x-\widetilde{x}^{\prime},
$$

which along with the weak closedness of $Q$ derives that $x-\tilde{x} \in Q$. Now, we only need to verify that

$$
\nabla f(\bar{p}, \bar{x})(p, \widetilde{x}) \in D^{w} \mathscr{F}(\bar{p}, \bar{y})(p)
$$

Setting

$$
y_{n}:=\frac{1}{t_{n}}\left(f\left(\bar{p}+t_{n} p_{n}, \bar{x}+t_{n} \widetilde{x}_{n}\right)-\bar{y}\right),
$$

we deduce from (3.3) that

$$
\bar{y}+t_{n} y_{n} \in \mathscr{F}\left(\bar{p}+t_{n} p_{n}\right) .
$$

Since $f$ is continuously Fréchet differentiable at $(\bar{p}, \bar{x})$, we obtain that

$$
f\left(\bar{p}+t_{n} p_{n}, \bar{x}+t_{n} \widetilde{x}_{n}^{\prime}\right)=f(\bar{p}, \bar{x})+t_{n} \nabla f(\bar{p}, \bar{x})\left(p_{n}, \widetilde{x}_{n}\right)+o\left(\left\|t_{n}\left(p_{n}, \widetilde{x}_{n}\right)\right\|\right) .
$$

From $\bar{y}+t_{n} y_{n}=f\left(\bar{p}+t_{n} p_{n}, \bar{x}+t_{n} \widetilde{x}_{n}^{\prime}\right)$ and $\bar{y}=f(\bar{p}, \bar{x})$, we deduce that

$$
y_{n}=\nabla f(\bar{p}, \bar{x})\left(p_{n}, \widetilde{x}_{n}\right)+\frac{1}{t_{n}} o\left(\left\|t_{n}\left(p_{n}, \widetilde{x}_{n}^{\prime}\right)\right\|\right) .
$$

Letting $n \rightarrow \infty$, we obtain $y_{n} \rightarrow \nabla f(\bar{p}, \bar{x})\left(p, \widetilde{x}^{\prime}\right)$. Hence, $y_{n} \underset{w}{\rightarrow} \nabla f(\bar{p}, \bar{x})(p, \widetilde{x})$. From this and (3.4), it confirms that

$$
\nabla f(\bar{p}, \bar{x})\left(p, \tilde{x}^{\prime}\right) \in D^{w} \mathscr{F}(\bar{p}, \bar{y})(p) .
$$

Hence,

$$
D^{w}(\mathscr{S}+Q)(\bar{p}, \bar{x})(p) \subseteq\left\{x \in X \mid x \in D^{w} C(\bar{p}, \bar{x})(p), \nabla f(\bar{p}, \bar{x})(p, x) \in D^{w} \mathscr{F}(\bar{p}, \bar{y})(p)\right\}+Q .
$$

Now, we prove the inversion of the above inclusion. Let $x \in D^{w} C(\bar{p}, \bar{x})(p)$ and

$$
y:=\nabla f(\bar{p}, \bar{x})(p, x) \in D^{w} \mathscr{F}(\bar{p}, \bar{y})(p) .
$$

Then, there exist $t_{n} \downarrow 0$ and $\left(p_{n}, y_{n}\right) \underset{s, w}{\longrightarrow}(p, y)$ such that

$$
\bar{y}+t_{n} y_{n} \in \mathscr{F}\left(\bar{p}+t_{n} p_{n}\right) \subset F\left(\bar{p}+t_{n} p_{n}\right) .
$$

Hence, there exists $x_{n} \in C\left(\bar{p}+t_{n} p_{n}\right)$ such that $\bar{y}+t_{n} p_{n}=f\left(\bar{p}+t_{n} p_{n}, x_{n}\right)$. Setting $\widetilde{x}_{n}:=\frac{1}{t_{n}}\left(x_{n}-\bar{x}\right)$, we get

$$
\bar{y}+t_{n} y_{n}=f\left(\bar{p}+t_{n} p_{n}, \bar{x}+t_{n} \widetilde{x}_{n}\right) .
$$

and

$$
x_{n}=\bar{x}+t_{n} \widetilde{x}_{n} \in C\left(\bar{p}+t_{n} p_{n}\right) .
$$

This, taking into account (ii), derives that $\widetilde{x}_{n} \underset{w}{\rightarrow} \widetilde{x}$. Thus, $\widetilde{x} \in D^{w} C(\bar{p}, \bar{x})(p)$. Moreover, since $f$ is Fréchet differentiable at $(\bar{p}, \bar{x})$, we obtain that

$$
f\left(\bar{p}+t_{n} p_{n}, \bar{x}+t_{n} \widetilde{x}_{n}\right)=f(\bar{p}, \bar{x})+t_{n} \nabla f(\bar{p}, \bar{x})\left(p_{n}, \widetilde{x}_{n}\right)+o\left(\left\|t_{n}\left(p_{n}, \widetilde{x}_{n}\right)\right\|\right) .
$$

From (3.7), (3.9) and $\bar{y}=f(\bar{p}, \bar{x})$, we deduce that

$$
y_{n}=\nabla f(\bar{p}, \bar{x})\left(p_{n}, \widetilde{x}_{n}\right)+\frac{o\left(\left\|t_{n}\left(p_{n}, \widetilde{x}_{n}\right)\right\|\right)}{t_{n}} .
$$


Letting $n \rightarrow \infty$, we obtain $y_{n} \rightarrow \nabla f(\bar{p}, \bar{x})(p, \widetilde{x})$, which leads to

$$
y_{n} \underset{w}{\rightarrow} \nabla f(\bar{p}, \bar{x})(p, \widetilde{x})=\nabla_{p} f(\bar{p}, \bar{x})(p)+\nabla_{x} f(\bar{p}, \bar{x})(\widetilde{x}) .
$$

Combining this with $y_{n} \underset{w}{\rightarrow} y=\nabla_{p} f(\bar{p}, \bar{x})(p)+\nabla_{x} f(\bar{p}, \bar{x})(x)$, one yields

$$
\nabla_{x} f(\bar{p}, \bar{x})(x)=\nabla_{x} f(\bar{p}, \bar{x})(\widetilde{x}) .
$$

From $\nabla_{x} f(\bar{p}, \bar{x})($.$) is strictly monotone on X$, we get $x=\widetilde{x}$. So, it follows from (3.6),(3.7) and (3.8) that there exist $t_{n} \downarrow 0$ and $\left(p_{n}, \widetilde{x}_{n}\right) \underset{s, w}{\longrightarrow}(p, x)$ and

$$
\bar{x}+t_{n} \widetilde{x}_{n} \in \mathscr{S}\left(\bar{p}+t_{n} p_{n}\right)
$$

i.e., $x \in D^{w} \mathscr{S}(\bar{p}, \bar{x})(p)$. Therefore,

$$
\left\{x \in X \mid x \in D^{w} C(\bar{p}, \bar{x})(p), \nabla f(\bar{p}, \bar{x})(p, x) \in D^{w} \mathscr{F}(\bar{p}, \bar{y})(p)\right\} \subseteq D^{w} \mathscr{S}(\bar{p}, \bar{x})(p) .
$$

Consequently,

$$
\left\{x \in X \mid x \in D^{w} C(\bar{p}, \bar{x})(p), \nabla f(\bar{p}, \bar{x})(p, x) \in D^{w} \mathscr{F}(\bar{p}, \bar{y})(p)\right\}+Q \subseteq D^{w} \mathscr{S}(\bar{p}, \bar{x})(p)+Q .
$$

By invoking Lemma 2.1, we obtain

$$
D^{w} \mathscr{S}(\bar{p}, \bar{x})(p)+Q \subset D^{w}(\mathscr{S}+Q)(\bar{p}, \bar{x})(p) .
$$

Therefore,

$$
\left\{x \in X \mid x \in D^{w} C(\bar{p}, \bar{x})(p), \nabla f(\bar{p}, \bar{x})(p, x) \in D^{w} \mathscr{F}(\bar{p}, \bar{y})(p)\right\}+Q \subseteq D^{w}(\mathscr{S}+Q)(\bar{p}, \bar{x})(p) .
$$

So, the claim (3.1) holds. From the definition of the generalized $\tau^{w}$-contingent epiderivative and (3.1), we have

$$
\begin{aligned}
& D_{g}^{w} \mathscr{S}(\bar{p}, \bar{x})(p) \\
& =\operatorname{Min}_{Q} D^{w}(\mathscr{S}+Q)(\bar{p}, \bar{x})(p) \\
& =\operatorname{Min}_{Q}\left\{\left\{x \in X \mid x \in D^{w} C(\bar{p}, \bar{x})(p), \nabla f(\bar{p}, \bar{x})(p, x) \in D^{w} \mathscr{F}(\bar{p}, \bar{y})(p)\right\}+Q\right\} \\
& =\operatorname{Min}_{Q}\left\{x \in X \mid x \in D^{w} C(\bar{p}, \bar{x})(p), \nabla f(\bar{p}, \bar{x})(p, x) \in D^{w} \mathscr{F}(\bar{p}, \bar{y})(p)\right\} .
\end{aligned}
$$

The proof is complete.

Now, we will give the formula for computing the generalized $\tau^{w}$ - contingent epiderivative of the frontier map $\mathscr{F}$.

Proposition 3.2. Let $\bar{p} \in P, \bar{x} \in C(\bar{p})$ and $\bar{y}=f(\bar{p}, \bar{x})$. Suppose that the following provisos hold:

(i) $f$ is continuously Fréchet differentiable at $(\bar{p}, \bar{x})$ with the derivative $\nabla f(\bar{p}, \bar{x})$;

(ii) $C$ is directionally compact at $(\bar{p}, \bar{x})$ in any direction $p \in X$;

(iii) The domination property holds for $F$ around $\bar{p}$.

Then,

$$
D_{g}^{w} \mathscr{F}(\bar{p}, \bar{y})(p)=\operatorname{Min}_{K}\left\{y \in Y \mid \exists x \in D^{w} C(\bar{p}, \bar{x})(p), y=\nabla f(\bar{p}, \bar{x})(p, x)\right\}, \forall p \in P .
$$

Proof. First, we verify that

$$
D^{w}(F+K)(\bar{p}, \bar{y})(p) \subseteq\left\{y \in Y \mid \exists x \in D^{w} C(\bar{p}, \bar{x})(p), y=\nabla f(\bar{p}, \bar{x})(p, x)\right\}+K, \forall p \in P .
$$

Let $y \in D^{w}(F+K)(\bar{p}, \bar{y})(p)$. Then, there exist $t_{n} \downarrow 0$ and $\left(p_{n}, y_{n}\right) \underset{s, w}{\longrightarrow}(p, y)$ such that

$$
\bar{y}+t_{n} y_{n} \in F\left(\bar{p}+t_{n} p_{n}\right)+K .
$$


This assures the existence of $x_{n} \in C\left(\bar{p}+t_{n} p_{n}\right)$ and $k_{n} \in K$ such that

$$
\bar{y}+t_{n} y_{n}=f\left(\bar{p}+t_{n} p_{n}, x_{n}\right)+k_{n} .
$$

Setting $x_{n}^{\prime}:=\frac{1}{t_{n}}\left(x_{n}-\bar{x}\right)$, we have

$$
\bar{y}+t_{n} y_{n}=f\left(\bar{p}+t_{n} p_{n}, \bar{x}+t_{n} x_{n}^{\prime}\right)+k_{n}
$$

and

$$
x_{n}=\bar{x}+t_{n} x_{n}^{\prime} \in C\left(\bar{p}+t_{n} p_{n}\right) .
$$

The above relation together with (ii) derives that $x_{n}^{\prime} \underset{w}{\rightarrow} x^{\prime}$. Thus, $x^{\prime} \in D^{w} C(\bar{p}, \bar{x})(p)$. It follows from (3.11) and $\bar{y}=f(\bar{p}, \bar{x})$ that

$$
y_{n}-\frac{f\left(\bar{p}+t_{n} p_{n}, \bar{x}+t_{n} x_{n}^{\prime}\right)-f(\bar{p}, \bar{x})}{t_{n}}=\frac{k_{n}}{t_{n}} \in K .
$$

Moreover, since $f$ is Fréchet differentiable at $(\bar{p}, \bar{x})$, we obtain that

$$
\frac{f\left(\bar{p}+t_{n} p_{n}, \bar{x}+t_{n} x_{n}^{\prime}\right)-f(\bar{p}, \bar{x})}{t_{n}} \rightarrow \nabla f(\bar{p}, \bar{x})\left(p, x^{\prime}\right) .
$$

As $K$ is a closed cone, one has $y-\nabla f(\bar{p}, \bar{x})\left(p, x^{\prime}\right) \in K$, and hence, $y \in \nabla f(\bar{p}, \bar{x})\left(p, x^{\prime}\right)+K$. It follows that (3.10) holds.

Now, we will prove that the inversion of (3.10) also holds. Let $p \in P$ and $y$ be in the right hand side of (3.10). This asserts the existence of $x \in D^{w} C(\bar{p}, \bar{x})(p)$ satisfying $y=\nabla f(\bar{p}, \bar{x})(p, x)$. Then, there exist $t_{n} \downarrow 0$ and $\left(p_{n}, x_{n}\right) \underset{s, w}{\longrightarrow}(p, x)$ such that $\bar{x}+t_{n} x_{n} \in C\left(\bar{p}+t_{n} p_{n}\right)$. Consequently,

$$
f\left(\bar{p}+t_{n} p_{n}, \bar{x}+t_{n} x_{n}\right) \in F\left(\bar{p}+t_{n} p_{n}\right), \forall n \in \mathbb{N} .
$$

Setting $y_{n}:=\frac{1}{t_{n}}\left(f\left(\bar{p}+t_{n} p_{n}, \bar{x}+t_{n} x_{n}\right)-\bar{y}\right)$, it follows that

$$
\bar{y}+t_{n} y_{n}=f\left(\bar{p}+t_{n} p_{n}, \bar{x}+t_{n} x_{n}\right) \in F\left(\bar{p}+t_{n} p_{n}\right), \forall n .
$$

Since $f$ is Fréchet differentiable at $(\bar{p}, \bar{x})$, one has

$$
f\left(\bar{p}+t_{n} p_{n}, \bar{x}+t_{n} x_{n}\right)=f(\bar{p}, \bar{x})+t_{n} \nabla f(\bar{p}, \bar{x})(p, x)+o\left(\left\|t_{n}\left(p_{n}, x_{n}\right)\right\|\right) .
$$

From the above equality and $\bar{y}=f(\bar{p}, \bar{x})$, we have

$$
y_{n}=\nabla f(\bar{p}, \bar{x})(p, x)+\frac{o\left(\left\|t_{n}\left(p_{n}, x_{n}\right)\right\|\right)}{t_{n}} .
$$

Letting $n \rightarrow \infty$, we obtain $y_{n} \rightarrow \nabla f(\bar{p}, \bar{x})(p, x)$. Thus, $y_{n} \underset{w}{\rightarrow} \nabla f(\bar{p}, \bar{x})(p, x)$. Combining this and (3.12), one has $y=\nabla f(\bar{p}, \bar{x})(p, x) \in D^{w} F(\bar{p}, \bar{y})(p)$ and

$$
\left\{y \in Y|y \in Y| \exists x \in D^{w} C(\bar{p}, \bar{x})(p), y=\nabla f(\bar{p}, \bar{x})(p, x)\right\} \subseteq D^{w} F(\bar{p}, \bar{y})(p) .
$$

It entails that

$$
\left\{y \in Y|y \in Y| \exists x \in D^{w} C(\bar{p}, \bar{x})(p), y=\nabla f(\bar{p}, \bar{x})(p, x)\right\}+K \subseteq D^{w} F(\bar{p}, \bar{y})(p)+K .
$$

Invoking Lemma 2.1, we obtain

$$
D^{w} F(\bar{p}, \bar{y})(p)+K \subset D^{w}(F+K)(\bar{p}, \bar{y})(p) .
$$

Therefore,

$$
\left\{y \in Y \mid \exists x \in D^{w} C(\bar{p}, \bar{x})(p), y=\nabla f(\bar{p}, \bar{x})(p, x)\right\}+K \subseteq D^{w}(F+K)(\bar{p}, \bar{y})(p),
$$


which along with (3.10) tells us that

$$
D^{w}(F+K)(\bar{p}, \bar{y})(p)=\left\{y \in Y \mid \exists x \in D^{w} C(\bar{p}, \bar{x})(p), y=\nabla f(\bar{p}, \bar{x})(p, x)\right\}+K, \forall p \in P .
$$

From the definition of the generalized $\tau^{w}$-contingent epiderivative, we derive from (3.13) that

$$
\begin{aligned}
D_{g}^{w} F(\bar{p}, \bar{y})(p) & =\operatorname{Min}_{K} D^{w}(F+K)(\bar{p}, \bar{y})(p) \\
& =\operatorname{Min}_{K}\left\{\left\{y \in Y \mid \exists x \in D^{w} C(\bar{p}, \bar{x})(p), y=\nabla f(\bar{p}, \bar{x})(p, x)\right\}+K\right\} \\
& =\operatorname{Min}_{K}\left\{y \in Y \mid \exists x \in D^{w} C(\bar{p}, \bar{x})(p), y=\nabla f(\bar{p}, \bar{x})(p, x)\right\} .
\end{aligned}
$$

On the other hand, since $\mathscr{F}(p) \subset F(p)$ for all $p \in P$ and the domination property holds for $F$ around $\bar{p}$, there exists a neighborhood $U \in \mathscr{N}(\bar{p})$ such that $\mathscr{F}(p)+K=F(p)+K, \forall p \in U$. Therefore,

$$
D^{w}(\mathscr{F}+K)(\bar{p}, \bar{y})(p)=D^{w}(F+K)(\bar{p}, \bar{y})(p), \forall p \in P,
$$

which in turn derives that, for each $p \in P$,

$$
D_{g}^{w} \mathscr{F}(\bar{p}, \bar{y})(p)=D_{g}^{w} F(\bar{p}, \bar{y})(p)=\operatorname{Min}_{K}\left\{y \in Y \mid \exists x \in D^{w} C(\bar{p}, \bar{x})(p), y=\nabla f(\bar{p}, \bar{x})(p, x)\right\} .
$$

The conclusion is obtained.

The following example illustrates the results in Proposition 3.1 and Proposition 3.2.

Example 3.1. [33] Let $P=X=Y=l^{2}, Q=K=l_{+}^{2}, f(p, x)=p+x$, and $C: l^{2} \rightrightarrows l^{2}$ be defined by

$$
C(p)= \begin{cases}\{x \in X \mid x \in p+K,\|x\| \leq 2\|p\|\} \cup\left\{2 p+p^{2}\right\}, & \text { if } p \in l_{+}^{2} \cap B(0,1), \\ \emptyset, & \text { otherwise. }\end{cases}
$$

Then,

$$
\begin{gathered}
F(p)= \begin{cases}\{y \in Y \mid y \in 2 p+K,\|y\| \leq 3\|p\|\} \cup\left\{3 p+p^{2}\right\}, & \text { if } p \in l_{+}^{2} \cap B(0,1), \\
\emptyset, & \text { otherwise, }\end{cases} \\
\mathscr{F}(p)=\left\{\begin{array}{ll}
\{2 p\}, & \text { if } p \in l_{+}^{2} \cap B(0,1), \\
\emptyset, & \text { otherwise, }
\end{array} \quad(\mathscr{F}+K)(p)= \begin{cases}\{2 p+K\}, & \text { if } p \in l_{+}^{2} \cap B(0,1), \\
\emptyset, & \text { otherwise. }\end{cases} \right.
\end{gathered}
$$

Taking $(\bar{p}, \bar{x})=(0,0)$, one has $\bar{y}=f(\bar{p}, \bar{x})=0$, and for every $p, x \in l^{2}$,

$$
\nabla f(\bar{p}, \bar{x})(p, x)=p+x .
$$

We can check that the assumptions (i), (ii) and (iii) in Proposition 3.2 are fulfilled. By direct calculations, one has

$$
\begin{gathered}
D^{w} C(\bar{p}, \bar{y})(p)= \begin{cases}\{x \in X \mid x \in p+K,\|x\| \leq 2\|p\|\}, & \text { if } p \in l_{+}^{2}, \\
\emptyset, & \text { otherwise, }\end{cases} \\
D^{w}(\mathscr{F}+K)(\bar{p}, \bar{y})(p)= \begin{cases}\{y \in Y \mid y \in 2 p+K\}, & \text { if } p \in l_{+}^{2}, \\
\emptyset, & \text { otherwise, }\end{cases} \\
D_{g}^{w} \mathscr{F}(\bar{p}, \bar{y})(p)= \begin{cases}\{2 p\}, & \text { if } p \in l_{+}^{2}, \\
\emptyset, & \text { otherwise, }\end{cases} \\
\left\{y \in Y \mid \exists x \in D^{w} C(\bar{p}, \bar{x})(p), y=\nabla f(\bar{p}, \bar{x})(p, x)\right\}
\end{gathered}
$$


Thus, all the assumptions in Proposition 3.2 are satisfied. So, for any $p \in l^{2}$,

$$
D_{g}^{w} \mathscr{F}(\bar{p}, \bar{y})(p)=\operatorname{Min}_{K}\left\{y \in Y \mid \exists x \in D^{w} C(\bar{p}, \bar{x})(p), y=\nabla f(\bar{p}, \bar{x})(p, x)\right\} .
$$

Moreover, we can check that

$$
\mathscr{S}(p)=\left\{\begin{array}{ll}
\{p\}, & \text { if } p \in l_{+}^{2} \cap B(0,1), \\
\emptyset, & \text { otherwise },
\end{array}(\mathscr{S}+Q)(p)= \begin{cases}\{p+Q\}, & \text { if } p \in l_{+}^{2} \cap B(0,1), \\
\emptyset, & \text { otherwise }\end{cases}\right.
$$

Hence,

$$
\begin{gathered}
D^{w}(\mathscr{S}+Q)(\bar{p}, \bar{x})(p)=\left\{\begin{array}{ll}
\{p+Q\}, & \text { if } p \in l_{+}^{2}, \\
\emptyset, & \text { otherwise, }
\end{array} D_{g}^{w} \mathscr{S}(\bar{p}, \bar{x})(p)= \begin{cases}\{p\}, & \text { if } p \in l_{+}^{2}, \\
\emptyset, & \text { otherwise, }\end{cases} \right. \\
\left\{x \in X \mid x \in D^{w} C(\bar{p}, \bar{x})(p), \nabla f(\bar{p}, \bar{x})(p, x) \in D^{w} \mathscr{F}(\bar{p}, \bar{y})(p)\right\}= \begin{cases}\{p\}, & \text { if } p \in l_{+}^{2}, \\
\emptyset, & \text { otherwise. }\end{cases}
\end{gathered}
$$

This leads that

$$
D_{g}^{w} \mathscr{S}(\bar{p}, \bar{x})(p)=\operatorname{Min}_{Q}\left\{x \in X \mid x \in D^{w} C(\bar{p}, \bar{x})(p), \nabla f(\bar{p}, \bar{x})(p, x) \in D^{w} \mathscr{F}(\bar{p}, \bar{y})(p)\right\} .
$$

4. The APPliCATION TO THE PARAMETRIC SEMI-INFINITE PROGRAMMING

In this section, we apply the results obtained in previous section to the problem $\left(\mathrm{PVO}_{p}\right)$ with the constraint mapping $C: P \rightrightarrows X$ defined by

$$
C(p):=\left\{x \in X \mid g_{t}(p, x) \leq 0, t \in T\right\},
$$

where $T$ is an arbitrary index set and, for each $t \in T, g_{t}: P \times X \rightarrow \mathbb{R}$ is continuously Fréchet differentiable maps. Constraints of type (4.1) are known as semi-infinite inequality constraints (see [10]). Denote $T(\bar{p}, \bar{x}):=\left\{t \in T \mid g_{t}(\bar{p}, \bar{x})=0\right\}$ the index set of all active constraints at $(\bar{p}, \bar{x})$.

Definition 4.1. Let $C$ be defined in (4.1) and let $(\bar{p}, \bar{x}) \in \operatorname{gr} C$. We say that $C$ satisfies the Abadie constraint qualification (ACQ) at $(\bar{p}, \bar{x})$ if

$$
T^{w}(\operatorname{gr} C,(\bar{p}, \bar{x})) \subseteq\left\{(p, x) \in P \times X \mid \nabla g_{t}(\bar{p}, \bar{x})(p, x) \leq 0, \forall t \in T(\bar{p}, \bar{x})\right\} .
$$

When $P, X$ are finite dimensional spaces, some sufficient conditions for (ACQ) could be established similarly to the Section 4 in [32].

The following proposition gives us a criterion for computing the $\tau^{w}$-contingent epiderivative of the constraint mapping $C$ in (4.1).

Proposition 4.1. Let $(\bar{p}, \bar{x}) \in g r C$. Suppose that $C$ in (4.1) satisfies (ACQ) in (4.2). Then

$$
D^{w} C(\bar{p}, \bar{x})(p)=\left\{x \in X \mid \nabla g_{t}(\bar{p}, \bar{x})(p, x) \leq 0, \forall t \in T(\bar{p}, \bar{x})\right\}, \forall p \in P .
$$

Proof. Let $p \in P$ and $x \in D^{w} C(\bar{p}, \bar{x})(p)$. Then, there exists $\tau_{n} \downarrow 0$ and $\left(p_{n}, x_{n}\right) \underset{s, w}{\longrightarrow}(p, x)$ such that

$$
\bar{x}+\tau_{n} x_{n} \in C\left(\bar{p}_{k}+\tau_{n} p_{n}\right), \forall n
$$

leads to

$$
g_{t}\left(\bar{p}+\tau_{n} p_{n}, \bar{x}+\tau_{n} x_{n}\right) \leq 0, \forall n, \forall t \in T .
$$

We deduce from the continuously Fréchet differentiability of $g_{t}$ that

$$
g_{t}(\bar{p}, \bar{x})+\tau_{n} \nabla g_{t}(\bar{p}, \bar{x})\left(p_{n}, x_{n}\right)+o\left(\tau_{n}\left\|\left(p_{n}, x_{n}\right)\right\|\right) \leq 0, \forall n, \forall t \in T .
$$


Since, $g_{t}(\bar{p}, \bar{x})=0$ for all $t \in T(\bar{p}, \bar{x})$, one has

$$
\nabla g_{t}(\bar{p}, \bar{x})\left(p_{n}, x_{n}\right)+\frac{1}{\tau_{n}} o\left(\tau_{n}\left\|\left(p_{n}, x_{n}\right)\right\|\right) \leq 0, \forall n, \forall t \in T(\bar{p}, \bar{x}) .
$$

Letting $n \rightarrow \infty$, the above inequality derives that

$$
\nabla g_{t}(\bar{p}, \bar{x})(p, x) \leq 0, \forall t \in T(\bar{p}, \bar{x}) .
$$

Hence,

$$
D^{w} C(\bar{p}, \bar{x})(p) \subseteq\left\{x \in X \mid \nabla g_{t}(\bar{p}, \bar{x})(p, x) \leq 0, \forall t \in T(\bar{p}, \bar{x})\right\}, \forall p \in P .
$$

This together with (ACQ) implies that

$$
D^{w} C(\bar{p}, \bar{x})(p)=\left\{x \in X \mid \nabla g_{t}(\bar{p}, \bar{x})(p, x) \leq 0, \forall t \in T(\bar{p}, \bar{x})\right\}, \forall p \in P .
$$

The proof is complete.

As the immediate consequences of Proposition 3.1, Proposition 3.2 and Proposition 4.1, we have the following results.

Proposition 4.2. Let $\bar{p} \in P$ and let $\bar{x} \in \mathscr{S}(\bar{p})$ be such that $\bar{y}=f(\bar{p}, \bar{x}) \in \mathscr{F}(\bar{p})$ with the constraint mapping $C$ given by (4.1). Suppose that the following conditions hold:

(i) $f$ is continuously Fréchet differentiable at $(\bar{p}, \bar{x})$ with derivative $\nabla f(\bar{p}, \bar{x})$;

(ii) $C$ satisfies $(A C Q)$ at $(\bar{p}, \bar{x})$;

(iii) $D^{w}(\mathscr{S}+Q)(\bar{p}, \bar{x})(p)$

$$
=\left\{x \in X \mid \nabla g_{t}(\bar{p}, \bar{x})(p, x) \leq 0, \forall t \in T(\bar{p}, \bar{x}), \nabla f(\bar{p}, \bar{x})(p, x) \in D^{w} \mathscr{F}(\bar{p}, \bar{y})(p)\right\}+Q .
$$

Then,

$D_{g}^{w} \mathscr{S}(\bar{p}, \bar{x})(p)=\operatorname{Min}_{Q}\left\{x \in X \mid \nabla g_{t}(\bar{p}, \bar{x})(p, x) \leq 0, \forall t \in T(\bar{p}, \bar{x}), \nabla f(\bar{p}, \bar{x})(p, x) \in D^{w} \mathscr{F}(\bar{p}, \bar{y})(p)\right\}$.

Proposition 4.3. Let $\bar{p} \in P$ and let $\bar{x} \in C(\bar{p})$ be such that $\bar{y}=f(\bar{p}, \bar{x}) \in \mathscr{F}(\bar{p})$ with the constraint mapping $C$ given by (4.1). Suppose that the following suppositions are fulfilled:

(i) the domination property holds for $F$ around $\bar{p}$;

(ii) $f$ is Fréchet differentiable at $(\bar{p}, \bar{x})$ with derivative $\nabla f(\bar{p}, \bar{x})$;

(iii) $C$ satisfies $(A C Q)$ at $(\bar{p}, \bar{x})$;

(iv) $D^{w}(F+K)(\bar{p}, \bar{y})(p)=\left\{y \in Y \mid \nabla g_{t}(\bar{p}, \bar{x})(p, x) \leq 0, \forall t \in T(\bar{p}, \bar{x}), y=\nabla f(\bar{p}, \bar{x})(p, x)\right\}+$ $K$.

Then,

$$
D_{g}^{w} \mathscr{F}(\bar{p}, \bar{y})(p)=\operatorname{Min}_{K}\left\{y \in Y \mid \nabla g_{t}(\bar{p}, \bar{x})(p, x) \leq 0, \forall t \in T(\bar{p}, \bar{x}), y=\nabla f(\bar{p}, \bar{x})(p, x)\right\} .
$$

The following example illustrates the results in Proposition 4.2 and Proposition 4.3.

Example 4.1. Let $T=[0,1] \cup\{-2\} \cup\{3\}, P=X=l^{2}, Y=\mathbb{R}^{2}, Q=l_{+}^{2}, K=\mathbb{R}_{+}^{2}$ and let $f$ : $l^{2} \times l^{2} \rightarrow \mathbb{R}^{2}, g_{t}: l^{2} \times l^{2} \rightarrow \mathbb{R}, t \in T$ be given as follows:

$$
\begin{gathered}
f(p, x):=\left(3 p_{1}+x_{1}, x_{2}\right), \forall p=\left(p_{1}, p_{2}, \ldots, p_{n}, \ldots\right) \in l^{2}, \forall x=\left(x_{1}, x_{2}, \ldots, x_{n}, \ldots\right) \in l^{2}, \\
g_{t}(p, x):=t p_{1}-t x_{1}+(t-1) x_{2}, \forall p, x \in l^{2} .
\end{gathered}
$$

We consider problem $\left(\mathrm{PVO}_{p}\right)$ with $C$ defined in (4.1). By some direct computations, we get

$$
\begin{aligned}
C(p) & =\left\{x \in l^{2} \mid-2 p_{1}+2 x_{1}-3 x_{2} \leq 0,3 p_{1}-3 x_{1}+2 x_{2} \leq 0\right\} \\
& =\left\{x \in l^{2} \mid x_{1} \geq p_{1}, x_{2} \geq 0\right\}, \forall p \in l^{2},
\end{aligned}
$$




$$
\begin{gathered}
F(p)=\left\{y \in \mathbb{R}^{2} \mid-8 p_{1}+2 y_{1}-3 y_{2} \leq 0,12 p_{1}-3 y_{1}+2 y_{2} \leq 0\right\} \\
=\left\{y \in \mathbb{R}^{2} \mid y_{1} \geq 4 p_{1}, y_{2} \geq 0\right\}, \forall p \in l^{2}, \\
\mathscr{F}(p)=\left\{\left(4 p_{1}, 0\right)\right\} .
\end{gathered}
$$

For $\bar{p}=0_{l^{2}}$, we have

$$
\begin{gathered}
C(\bar{p})=\left\{x \in l^{2} \mid x_{1} \geq 0, x_{2} \geq 0\right\} \\
F(\bar{p})=\left\{y \in \mathbb{R}^{2} \mid y_{1} \geq 0, y_{2} \geq 0\right\}, \mathscr{F}(\bar{p})=\{(0,0)\} .
\end{gathered}
$$

Thus, $\bar{x}=0_{l^{2}} \in C(\bar{p})$ and $\bar{y}=f(\bar{p}, \bar{x})=(0,0) \in \mathscr{F}(\bar{p})$. We can check that assumption (i) of Proposition 4.3 holds. For each $t \in T$, one has $T(\bar{p}, \bar{x})=T$,

$$
\begin{gathered}
T^{w}(\operatorname{gr} C,(\bar{p}, \bar{x}))=\left\{(p, x) \in l^{2} \times l^{2} \mid t p_{1}-t x_{1}-(1-t) x_{2} \leq 0, \forall t \in T\right\} \\
=\left\{(p, x) \in l^{2} \times l^{2} \mid x_{1} \geq p_{1}, x_{2} \geq 0\right\}, \\
\nabla g_{t}(\bar{p}, \bar{x})=\left(\nabla_{p} g_{t}(\bar{p}, \bar{x}), \nabla_{x} g_{t}(\bar{p}, \bar{x})\right)=((t, 0, \ldots, 0, \ldots),(-t, t-1,0, \ldots, 0, \ldots)), t \in T, \\
\left\{(p, x) \in P \times X \mid \nabla g_{t}(\bar{p}, \bar{x})(p, x) \leq 0, \forall t \in T(\bar{p}, \bar{x})\right\}=\left\{(p, x) \in l^{2} \times l^{2} \mid x_{1} \geq p_{1}, x_{2} \geq 0\right\},
\end{gathered}
$$

which implies that $C$ satisfies (ACQ) at $(\bar{p}, \bar{x})$. Moreover, for any $p \in l^{2}$,

$$
\begin{gathered}
D^{w}(F+K)(\bar{p}, \bar{y})(p)=D^{w} F(\bar{p}, \bar{y})(p)=\left\{y \in \mathbb{R}^{2} \mid y_{1} \geq 4 p_{1}, y_{2} \geq 0\right\}, \\
\nabla f(\bar{p}, \bar{x})=\left(\nabla_{p} f(\bar{p}, \bar{x}), \nabla_{x} f(\bar{p}, \bar{x})\right) \\
=(((3,0, \ldots, 0, \ldots),(0,0, \ldots, 0, \ldots)),((1,0, \ldots, 0, \ldots),(0,1,0, \ldots, 0, \ldots))), \\
\nabla f(\bar{p}, \bar{x})(p, x)=\left(3 p_{1}+x_{1}, x_{2}\right) .
\end{gathered}
$$

Thus, for any $p \in l^{2}$,

$$
D^{w}(F+K)(\bar{p}, \bar{y})(p)=\left\{y \in \mathbb{R}^{2} \mid \nabla g_{t}(\bar{p}, \bar{x})(p, x) \leq 0, \forall t \in T(\bar{p}, \bar{x}), y=\nabla f(\bar{p}, \bar{x})(p, x)\right\}+K,
$$

i.e., all assumptions of Proposition 4.3 are satisfied. Since $D^{w} \mathscr{F}(\bar{p}, \bar{y})(p)=\left\{\left(4 p_{1}, 0\right)\right\}$, one has

$$
D_{g}^{w} \mathscr{F}(\bar{p}, \bar{y})(p)=\operatorname{Min}_{K}\left\{y \in \mathbb{R}^{2} \mid \nabla g_{t}(\bar{p}, \bar{x})(p, x) \leq 0, \forall t \in T(\bar{p}, \bar{x}), y=\nabla f(\bar{p}, \bar{x})(p, x)\right\},
$$

which derives that the conclusion of Proposition 4.3 holds.

Now, we will illuminate the results in Proposition 4.2. From the above analysis, one has that the assumptions (i), (ii) of Proposition 4.2 are fulfilled. Straightforward calculations give us

$$
\begin{gathered}
\mathscr{S}(p)=\left\{x \in l^{2} \mid x_{1}=p_{1}, x_{2}=0\right\}, \\
(\mathscr{S}+Q)(p)=\left\{x \in l^{2} \mid x_{1} \geq p_{1}, x_{2} \geq 0\right\}, \\
D^{w}(\mathscr{S}+Q)(p)=\left\{x \in l^{2} \mid x_{1} \geq p_{1}, x_{2} \geq 0\right\}, \\
\left\{x \in l^{2} \mid \nabla g_{t}(\bar{p}, \bar{x})(p, x) \leq 0, \forall t \in T(\bar{p}, \bar{x}), \nabla f(\bar{p}, \bar{x})(p, x) \in D^{w} \mathscr{F}(\bar{p}, \bar{y})(p)\right\} \\
=\left\{x \in l^{2} \mid x_{1}=p_{1}, x_{2}=0\right\} .
\end{gathered}
$$

Hence, for all $p \in l^{2}$,

$$
\begin{aligned}
& D^{w}(\mathscr{S}+Q)(\bar{p}, \bar{x})(p) \\
& =\left\{x \in l^{2} \mid \nabla g_{t}(\bar{p}, \bar{x})(p, x) \leq 0, \forall t \in T(\bar{p}, \bar{x}), \nabla f(\bar{p}, \bar{x})(p, x) \in D^{w} \mathscr{F}(\bar{p}, \bar{y})(p)\right\}+Q,
\end{aligned}
$$

i.e., assumption (iii) of Proposition 4.2 holds. As $D^{w} \mathscr{S}(\bar{p}, \bar{x})(p)=\left\{x \in l^{2} \mid x_{1}=p_{1}, x_{2}=0\right\}$, one gets

$$
\begin{aligned}
& D_{g}^{w} \mathscr{S}(\bar{p}, \bar{x})(p) \\
& =\operatorname{Min}_{Q}\left\{x \in l^{2} \mid \nabla g_{t}(\bar{p}, \bar{x})(p, x) \leq 0, \forall t \in T(\bar{p}, \bar{x}), \nabla f(\bar{p}, \bar{x})(p, x) \in D^{w} \mathscr{F}(\bar{p}, \bar{y})(p)\right\} .
\end{aligned}
$$


which confirms the conclusion of Proposition 4.2.

\section{REFERENCES}

[1] N.L.H. Anh, Some results on sensitivity analysis in set-valued optimization, Positivity 21 (2017), 1527-1543.

[2] J.P. Aubin, H. Frankowska, Set-valued Analysis, Birkäuser, Basel, 1990.

[3] E.M. Bednarczuk, W. Song, Contingent epiderivative and its applications to set-valued maps, Control Cyber. 27 ( 1998), 375-386.

[4] G.Y. Chen, J. Jahn, Optimality conditions for set-valued optimization problems, Math. Methods Oper. Res. 48 (1998), 187-200.

[5] T.D. Chuong, J. C. Yao, Generalized Clarke epiderivatives of parametric vector optimization problems, J. Optim. Theory Appl. 147 (2010), 77-94.

[6] T.D. Chuong, Clarke coderivatives of efficient point multifunctions in parametric vector optimization, Nonlinear Anal. 74 (2011), 273-285.

[7] T.D. Chuong, Derivatives of the efficient point multifunction in parametric vector optimization problems, J. Optim. Theory Appl. 156 (2013), 247-265.

[8] T.D. Chuong, J. C. Yao, Fréchet subdifferentials of efficient point multifunctions in parametric vector optimization, J. Global Optim. 57 (2013), 1229-1243.

[9] H.T.H. Diem, P.Q. Khanh, L.T. Tung, On higher-order sensitivity analysis in nonsmooth vector optimization, J. Optim.Theory Appl. 162 (2014), 463-488.

[10] M. A. Goberna, M. A. López, Linear Semi-Infinite Optimization, Wiley, Chichester, 1998.

[11] N.Q. Huy, G.M. Lee, On sensitivity analysis in vector optimization, Taiwaness J. Math. 11 (2007), 945-958.

[12] J. Jahn, R. Rauh, Contingent epiderivatives and set valued optimization, Math. Methods Oper. Res. 46 (1997) 193- 211.

[13] H. Kuk, T.Tanino, M. Tanaka, Sensitivity analysis in vector optimization, J. Optim. Theory Appl. 89 (1996), 713-730.

[14] G.M. Lee, N.Q. Huy, Sensitivity of solutions to a parametric generalized equation, Set-Valued Anal. 16 (2008), 805-820.

[15] D.T. Luc, M. Soleimani-damaneh, M. Zamani, Semi-differentiability of the marginal mapping in vector optimization, SIAM J. Optim. 28 (2018), 1255-1281.

[16] B.S. Mordukhovich, Variational Analysis and Generalized Differentiation. I: Basic Theory, Springer, Berlin, 2006.

[17] B.S. Mordukhovich, Variational Analysis and Generalized Differentiation. II: Application, Springer, Berlin, 2006.

[18] B.S. Mordukhovich, N.M. Nam, N.D. Yen, Subgradients of marginal functions in parametric mathematical programming, Math. Program. 116 (2009), 369-396.

[19] Z.H. Peng, Z.P. Wan, W.Z. Xiong, Sensitivity analysis in set-valued optimization under strictly minimal efficiency, Evol. Equ. Control Theory 6 (2017), 427-436.

[20] R.T. Rockafellar, R.J.-B. Wets, Variational Analysis, Springer, Berlin, 2009.

[21] R.T. Rockafellar, Proto-differentiablility of set-valued mappings and its applications in optimization, Ann. Inst. H. Poincaré Anal. Non Linéaire. 6 (1989), 449-482.

[22] L. Rodrígues-Marín, M. Sama, $\tau^{w}$-contingent epiderivatives in reflexive spaces, Nonlinear Anal. 68 (2008), 3780-3788.

[23] M. Sama, Some remarks on the existence and computation of contingent epiderivatives, Nonlinear Anal. 71 (2009), 2997-3007.

[24] M. Sama, The role of directional compactness in the existence and computation of contingent epiderivatives, J. Math. Anal. Appl. 372 (2010), 262-272.

[25] Y. Sawaragi, H. Nakayama, T. Tanino, Theory of Multiobjective Optimization, Academic Press, New York, 1985.

[26] D.S. Shi, Contingent derivative of the perturbation map in multiobjective optimization, J. Optim. Theory Appl. 70 (1991), 385-396. 
[27] X.K. Sun, S.J. Li, Generalized second-order contingent epiderivatives in parametric vector optimization problem, J. Global Optim. 58 (2014), 351-363.

[28] T. Tanino, Sensitivity analysis in multiobjective optimization, J. Optim. Theory Appl. 56 (1988), 479-499.

[29] L.T. Tung, Variational sets and asymptotic variational sets of proper perturbation map in parametric vector optimization, Positivity. 21 (2017), 1647-1673.

[30] L.T. Tung, On second-order proto-differentiability of perturbation maps, Set-Valued Var. Anal. 26 (2018), 561-579.

[31] L.T. Tung, On higher-order proto-differentiability of perturbation maps. Positivity (2019), doi: 10.1007/ s11117-019-00689-x.

[32] L.T. Tung, Karush-Kuhn-Tucker optimality conditions and duality for multiobjective semi-infinite programming via tangential subdifferential, Numer. Funct. Anal. Optim. (2019), doi: 10.1080/01630563.2019. 1667826 .

[33] L.T. Tung, P. T. Hung, Sensitivity analysis in parametric vector optimization in Banach spaces via $\tau^{w}$ contingent derivatives, Turk. J. Math (2019), doi: 10.3906/mat-1902-57. 